BULLETIN OF THE

AMERICAN MATHEMATICAL SOCIETY

Volume 77, Number 3, May 1971

\title{
SOME FIXED POINT THEOREMS
}

\author{
BY ROGER D. NUSSBAUM ${ }^{1}$
}

Communicated by Philip Hartman, October 23, 1970

Introduction. We wish to summarize here some new asymptotic fixed point theorems. By an asymptotic fixed point theorem we mean roughly a theorem in functional analysis in which the existence of fixed points of a map $f$ is proved with the aid of assumptions on the iterates $f^{n}$ of $f$. Such theorems have proved of use in the theory of ordinary and functional differential equations (see [7], [8], [9] and [15]). It also seems likely that many of the fixed point theorems which have been used in nonlinear functional analysis can be unified by obtaining them as corollaries of general asymptotic fixed point theorems. These theorems also give new results, of course.

In our first section we restrict attention to continuous maps $f$ defined on closed, convex subsets of Banach spaces. We obtain a general fixed point theorem (Theorem 1 below), and we prove that certain fixed point theorems of R. L. Frum-Ketkov [5], F. E. Browder [1], [2], W. A. Horn [6], G. Darbo [3], the author [11], [12], [13] and others follow as corollaries. In the second section we consider maps defined on more general spaces than closed, convex subsets of Banach spaces, and we generalize some of the results of $\S 1$.

1. We begin by recalling some notation from [11]. If $U$ is a closed subset of a Banach space $X$, we shall say that $U \in \mathcal{F}$ if there exists a closed, locally finite covering $\left\{C_{j}\right\}_{j \in J}$ of $U$ by closed, convex sets $C_{j} \subset U \subset X$. We shall say that $U \in \mathscr{F}_{0}$ if there exists a finite number of closed, convex sets $C_{1}, C_{2}, \cdots, C_{n}$ in $X$ such that $U=\bigcup_{j=1}^{n} C_{j}$.

The basic lemma in all our work is the following geometrical result, which can be viewed as a generalization of a theorem of Dugundji [4]. If $X$ below is a locally convex topological vector space, the same conclusions hold, with the exception that $R$ may not be a retraction.

Lemma 1. Let $C$ and $D$ be closed subsets of a Banach space $X$ with $C \supset D$. Assume that $C=\bigcup_{j=1}^{n} C_{j}$ and $D=\bigcup_{j=1}^{n} D_{j}$, where $C_{j} \supset D_{j}$ and $C_{j}$ and $D_{j}$ are closed, convex subsets of $X$ for $1 \leqq j \leqq n$. Suppose that, for each subset $J \subset\{1,2, \cdots, n\}, \cap_{j \in J} C_{j}$ is nonempty if and only if $\bigcap_{j \in J} D_{j}$

AMS 1969 subject classifications. Primary 4785, 4780; Secondary 5485.

Key words and phrases. Fixed points, asymptotic fixed point theorems, fixed point index, generalized Lefschetz number.

1 Partially supported by NSF GP 20228.

Copyright @ 1971, American Mathematical Society 
is nonempty. Then there exists a continuous retraction $R: C \rightarrow D$ such that $R\left(C_{j}\right) \subset C_{j}$ for $1 \leqq j \leqq n$.

Theorem 1. Let $G$ be a closed, convex subset of a Banach space $X$ and $f: G \rightarrow G$ a continuous map. Assume there exist a sequence of nonempty sets $\left\{U_{m}: 1 \leqq m<\infty\right\}, U_{m} \subset G$ for $1 \leqq m<\infty$, a sequence of nonnegative real numbers $\left\{r_{m}\right\}$ such that $\lim _{m \rightarrow \infty} r_{m}=0$, and a nonempty compact set $M \subset X$ such that the following conditions hold:

(1) $U_{m} \in F_{0}$ and $f\left(U_{m}\right) \subset U_{m}$ for $1 \leqq m<\infty$.

(2) $U_{m} \subset N_{r_{m}}(M)=\left\{x \in G: d(x, M)<r_{m}\right\}$.

(3) Given any compact set $A \subset G$ and any $U_{m}, 1 \leqq m<\infty$, there exists an integer $N$ (depending on $A$ and $U_{m}$ ) such that $f^{N}(A) \subset U_{m}$.

Then $\Lambda_{\text {gen }}\left(f \mid U_{m}\right)$, Leray's generalized Lefschetz number for $f$ restricted to $U_{m}$ (see [10]), is nonzero for all $m$, and $f$ has a fixed point.

The proof runs roughly as follows. By using Lemma 1 and some elementary facts about the generalized Lefschetz number, one proves that $\Lambda_{\text {gen }}\left(f \mid U_{m}\right) \neq 0$. Since $U_{m} \in \mathscr{F}_{0}$ and since $U_{m} \subset N_{r_{m}}(M)$, one proves that $U_{m}=\bigcup_{i=1}^{n(m)} C_{i, m}$, where $C_{i, m}$ are closed convex sets of diameter less than or equal to $s_{m}$ and $\lim _{m \rightarrow \infty} s_{m}=0$. By using Lemma 1 , one proves that there exists a continuous map $R_{m}: U_{m} \rightarrow U_{m}$ such that the range of $R_{m}$ lies in a finite-dimensional subspace $F_{m}$ of $X$ and $R\left(C_{i, m}\right)$ $\subset C_{i, m}$ for $1 \leqq i \leqq n(m)$. Since $R_{m} f$ and $f$ are homotopic in $U_{m}$, $\Lambda_{\text {gen }}\left(R_{m} f \mid U_{m}\right) \neq 0$. If $K_{m}=U_{m} \cap F_{m}$, which is a compact, metric ANR, this implies that $\Lambda_{\text {gen }}\left(R_{m} f \mid K_{m}\right) \neq 0$, and since the ordinary Lefschetz fixed point theorem applies to $K_{m}, R_{m} f$ has a fixed point $x_{m} \in K_{m}$. One proves that $\left\|f\left(x_{m}\right)-x_{m}\right\| \leqq s_{m}$. Since $d\left(x_{m}, M\right) \leqq r_{m}, x_{m}$ has a convergent subsequence approaching some point $x \in M \cap G$, and clearly $f(x)=x$.

Our first corollary was obtained by F. E. Browder (see [1, Theorem 16.3]) for the case of Hilbert spaces. Browder's proof does not seem to generalize directly to Banach spaces.

Corollary 1. Let $G$ be a closed, convex subset of a Banach space $X$ and $f: G \rightarrow G$ a continuous map. Assume that there exists a compact set $M \subset X$ and two sequences of positive numbers $\left\{a_{k}\right\}$ and $\left\{b_{k}\right\}$ with $a_{k}>b_{k}$ and $\lim _{k \rightarrow \infty} a_{k}=0$ such that

(1) for each open neighborhood $W$ of $M$ in $X$ and each $x \in G$, there exists an integer $N$ (depending on $x$ and $W$ ) such that $f^{n}(x) \in W$ for $n \geqq N$;

(2) f maps $N_{a_{k}}(M)=\left\{x \in G: d(x, M)<a_{k}\right\}$ into $N_{b_{k}}(M)$ for all $k \geqq 1$. Then $f$ has a fixed point.

Proof. For each $m \geqq 1$, let $x_{i, m}$ be an $\left(a_{m}-b_{m}\right) / 2$ net for $M, 1 \leqq i$ $\leqq n(m)$. Let $U_{i, m}=\left\{x \in G:\left\|x-x_{i, m}\right\| \leqq a_{m}\right\}$ and let $U_{m}=\bigcup_{i=1}^{n(m)} U_{i, m}$. 
It is not hard to check that all the conditions of Theorem 1 hold with $M$ and $\left\{U_{m}\right\}$ as above.

Our next corollary was claimed by R. L. Frum-Ketkov [5] for the case that $G$ is a closed ball in a Banach space, but Frum-Ketkov's proof appears to be incorrect. A correct proof for the case of a closed ball in a so-called $\pi_{1}$-space was given in [12] and [13].

Corollary 2. Let $G$ be a closed, convex subset of a Banach space $X$ and $f: G \rightarrow G$ a continuous map. Assume that there exists a compact, nonempty set $M \subset X$ and a constant $k<1$ such that $d(f(x), M)$ $\leqq k d(x, M)$ for all $x \in G$. Then $f$ has a fixed point.

Proof. This is a very special case of Corollary 1.

Corollary 3. Let $G$ be a closed, convex subset of a Banach space $X$ and $f: G \rightarrow G$ a continuous map. Assume that there exists a compact, nonempty set $M \subset G$ such that:

(1) $f(M) \subset M$.

(2) Given any compact set $A \subset G$ and any open neighborhood $W$ of $M$, there exists an integer $N$ (depending on $A$ and $W$ ) such that $f^{n}(A) \subset W$ for $n \geqq N$.

(3) There exists an open neighborhood $V$ of $M$ such that $f \mid V$ is a $k$-set-contraction $k<1$. (See [11] for definitions.)

Then $i_{G}(f, V)$, the generalized fixed point index defined in [11], is nonzero, and $f$ has a fixed point.

Corollary 3 follows from Theorem 1, but the argument is more involved than for Corollary 1. Corollary 3 immediately implies a theorem of G. Darbo [3] and the final two theorems given in [11].

Before stating our next corollary, we recall some further notation. If $G$ is a topological space, $f: G \rightarrow G$ a map, and $A$ a subset of $G$, the orbit of $A$ under $f, O(A)$, is $\bigcup_{j \geq 0} f^{j}(A)\left(f^{0}(A)=A\right)$. If $f$ and $G$ are as above, we write $C_{\infty}(f, G)=\bigcap_{n \geq 1} f^{n}(G)$.

Corollary 4. Let $G$ be a closed, convex subset of a Banach space $X$ and $f: G \rightarrow G$ a continuous map. Assume that

(1) $C_{\infty}(f, G)$ has compact closure.

(2) If $A$ is any compact subset of $G$, the orbit of $A$ has compact closure.

(3) There exists an open neighborhood $V$ of $\operatorname{cl}\left(C_{\infty}(f, G)\right)$ such that $f \mid V$ is a $k$-set-contraction, $k<1$.

Then $i_{G}(f, V) \neq 0$ and $f$ has a fixed point.

Proof. One can prove this follows from Corollary 3 with $M$ $=\operatorname{cl}\left(C_{\infty}(f, G)\right)$. 
Our next corollary is obtained by F. E. Browder in [1] and in less generality by $\mathrm{H}$. Steinlein [14].

Corollary 5 (Browder [1]). Let $G$ be a closed, convex subset of a Banach space $X$ and $f: G \rightarrow G$ a continuous map. Assume that

(1) $C_{\infty}(f, G)$ has compact closure.

(2) For each point $x \in G$, the orbit of $x$ has compact closure.

(3) There exists an open neighborhood $V$ of $\operatorname{cl}\left(C_{\infty}(f, G)\right.$ ) such that $f(V)$ has compact closure.

Then $i_{G}(f, V) \neq 0$ and $f$ has a fixed point.

Proof. With the aid of some lemmas of A. Gleason and R. S. Palais (unpublished) one can prove that under the above hypotheses the orbit of a compact set in $G$ has compact closure. Corollary 5 now follows from Corollary 4.

Corollary 6 (W. A. Horn [6]). Let $G$ be a closed, convex subset of a Banach space $X$ and $f: G \rightarrow G$ a compact map ( $f$ is continuous and takes bounded sets into precompact sets). Assume there exists a bounded set $E$ such that for each $x \in G$ there exists an integer $m(x)=m$ such that $f^{m}(x) \in E$. Then $i_{G}(f, G) \neq 0$ and $f$ has a fixed point.

Corollary 6 also holds for $f$ a $k$-set-contraction, $k<1$, with the added assumption that $f(E) \subset E$. For $f$ compact this assumption is unnecessary.

2. In this section we state some fixed point theorems for maps defined in spaces $G \in \mathcal{F}$. Our results here are more tentative than in $\$ 1$.

Before stating our second theorem, recall that a topological space $K$ is contractible in itself to a point if there exists $x_{0} \in K$ and a continuous map $F: K \times[0,1] \rightarrow K$ such that $F(x, 0)=x$ and $F(x, 1)=x_{0}$ for all $x \in K$.

Theorem 2. Suppose that $G \in F, U$ is an open subset of $G$ and $f: U \rightarrow U$ is a continuous map. Let $\rho:[0, a] \rightarrow[0, a](a>0)$ be $a$ decreasing real-valued map which is continuous from the right and is such that $\rho(r)<r$ for $0<r \leqq a$. A ssume also

(1) there exists a compact set $M \subset U$ such that

$$
N_{a}(M)=\{x \in G: d(x, N)<a\} \subset U
$$

and $f\left(N_{r}(M)\right) \subset N_{\rho(r)}(M)$ for $0 \leqq r \leqq a$.

(2) If $W$ is any open neighborhood of $M$ and $x \in G$, there exists an integer $N$ (depending on $x$ and $W$ ) such that $f^{N}(x) \in W$. 
(3) There exists a compact set $K \in F_{0}$ such that $M \subset K \subset U$ and such that $K$ is contractible in itself to a point.

Then $f$ has a fixed point.

A number of generalizations of Corollary 2 follow trivially from Theorem 2 , using $\rho(r)=k r, k<1$.

Theorem 3. Suppose that $G \in \mathcal{F}, U$ is an open subset of $G$ and $f: U \rightarrow U$ is a continuous map. Assume that there exists a compact set $M \subset U$ for which if $A$ is any compact subset of $U$ and $W$ any open neighborhood of $M$, there exists an integer $N$ such that $f^{n}(A) \subset W$ for $n \geqq N$. Assume that there exists an open neighborhood $V$ of $M$ such that $f \mid V$ is a $k$-set-contraction, $k<1$. Finally suppose that there exists a compact set $K \in F_{0}$ such that $M \subset K \subset U$ and $K$ is contractible in itself. Then $i_{G}(f, V) \neq 0$ and $f$ has a fixed point.

Theorem 3 is closely related to results in [11], [12] and [13], but it does not appear directly comparable.

The usual assumption above that $G \in \mathcal{F}$ is unnecessarily restrictive. It suffices that $G$ is a complete metric space and, for each $c>1$, there exist an isometric imbedding of $G$ into $G_{c} \in \mathcal{F}$ and a retraction $r_{c}$ of some open neighborhood $U_{c}$ of $j_{c}(G)$ on to $j_{c}(G)$ such that $r_{c}$ is a $c$-setcontraction. The following simple proposition is an application of this observation.

Propositron. Let $X$ be an infinite-dimensional Banach space and $S=\{x:\|x\|=1\}$. Then if $f: S \rightarrow S$ is a $k$-set-contraction, $k<1, f$ has a fixed point.

\section{REFERENCES}

1. F. E. Browder, Nonlinear operators and nonlinear equations of evolution, Proc. Sympos. Pure Math., vol. 18, part 2, Amcr. Math. Soc., Providence, R. I., 1970.

2. - Asymptotic fixed point theorems, Math. Ann. 185 (1970), 38-61.

3. G. Darbo, Punti uniti in trasformazioni a condominio non compatto, Rend. Sem. Mat. Univ. Padova 24 (1955), 84-92. MR 16, 1140.

4. J. Dugundji, An extension of Tietze's theorem, Pacific J. Math. 1 (1951), 353-367. MR 13,373.

5. R. L. Frum-Ketkov, Mappings into a Banach space sphere, Dokl. Akad. Nauk SSR 175 (1967), 1229-1231 = Soviet Math. Dokl. 8 (1967), 1004-1007. MR 36 \#3181.

6. W. A. Horn, Some fixed point theorems for compact maps and flows in Banach spaces, Trans. Amer. Math. Soc. 149 (1970), 391-404.

7. G. S. Jones, Asymptotic fixed point theorems and periodic systems of functionaldifferential equations, Contributions to Differential Equations 2 (1963), 385-405. MR $28 \# 1361$.

8. - Stability and asymptotic fixed point theory, Proc. Nat. Acad. Sci. U.S.A. 53 (1965), 1262-1264. 
9. M. A. Krasnosel'skir, The operator of translation along the trajectories of differential equations, "Nauka," Moscow, 1966; English transl., Transl. Math. Monographs, vol. 19, Amer. Math. Soc., Providence, R. I., 1968. MR 34 \#3012; MR 36 \#6688.

10. J. Leray, Theorie des points fixes, indice total et nombre de Lefschetz, Bull. Soc. Math. France 87 (1959), 221-233. MR $26 \# 762$.

11. R. D. Nussbaum, The fixed point index and asymptotic fixed point theorems for $k$-set-contractions, Bull. Amer. Math. Soc. 75 (1969), 490-495. MR 39 \#7589.

12. - The fixed point index and fixed point theorems for $k$-set-contractions, Ph.D. Dissertation, University of Chicago, Chicago, Ill., 1969 (unpublished).

13. —, Asymptotic fixed point theorems for local condensing maps, Math. Ann. (to appear).

14. H. Steinlein, Zur Existenz von Fixpunkten bei Abbildungen mit vollstetigen Iterierten, Ph.D. Dissertation, University of Munich, 1970.

15. T. Yoshizawa, Stable sets and periodic solutions in a perturbed system, Contributions to Differential Equations 2 (1963), 407-420. MR 28 \#1362.

Rutgers University, New Brunswick, New Jersey 08903 